\title{
Relativistic Néel-Order Fields Induced by Electrical Current in Antiferromagnets
}

\author{
J. Železný, ${ }^{1,2}$ H. Gao, ${ }^{3}$ K. Výborný, ${ }^{1}$ J. Zemen ${ }^{4}$ J. Mašek, ${ }^{5}$ Aurélien Manchon, ${ }^{6}$ J. Wunderlich, ${ }^{1,7}$ \\ Jairo Sinova, ${ }^{8,1,3}$ and T. Jungwirth ${ }^{1,9}$ \\ ${ }^{1}$ Institute of Physics ASCR, Cukrovarnická 10, 16253 Praha 6, Czech Republic \\ ${ }^{2}$ Faculty of Mathematics and Physics, Charles University in Prague, Ke Karlovu 3, 12116 Prague 2, Czech Republic \\ ${ }^{3}$ Department of Physics, Texas A\&M University, College Station, Texas 77843-4242, USA \\ ${ }^{4}$ Department of Physics, Blackett Laboratory, Imperial College London, London SW7 2AZ, United Kingdom \\ ${ }^{5}$ Institute of Physics ASCR, Na Slovance 2, 18221 Praha 8, Czech Republic \\ ${ }^{6}$ Physical Science and Engineering Division, King Abdullah University of Science and Technology (KAUST), \\ Thuwal 23955-6900, Kingdom of Saudi Arabia \\ ${ }^{7}$ Hitachi Cambridge Laboratory, Cambridge CB3 OHE, United Kingdom \\ ${ }^{8}$ Institut für Physik, Johannes Gutenberg Universität Mainz, 55128 Mainz, Germany \\ ${ }^{9}$ School of Physics and Astronomy, University of Nottingham, Nottingham NG7 2RD, United Kingdom \\ (Received 2 June 2014; revised manuscript received 13 August 2014; published 6 October 2014)
}

\begin{abstract}
We predict that a lateral electrical current in antiferromagnets can induce nonequilibrium Néel-order fields, i.e., fields whose sign alternates between the spin sublattices, which can trigger ultrafast spin-axis reorientation. Based on microscopic transport theory calculations we identify staggered current-induced fields analogous to the intraband and to the intrinsic interband spin-orbit fields previously reported in ferromagnets with a broken inversion-symmetry crystal. To illustrate their rich physics and utility, we consider bulk $\mathrm{Mn}_{2} \mathrm{Au}$ with the two spin sublattices forming inversion partners, and a $2 \mathrm{D}$ square-lattice antiferromagnet with broken structural inversion symmetry modeled by a Rashba spin-orbit coupling. We propose an antiferromagnetic memory device with electrical writing and reading.
\end{abstract}

DOI: 10.1103/PhysRevLett.113.157201

PACS numbers: 75.50.Ee, 75.47.- $\mathrm{m}$, 85.80.Jm

Commercial spin-based memory and storage devices rely on one type of magnetic order, ferromagnetism, and one basic principle, that the opposite spin orientations in a ferromagnet (FM) represent the $0 \mathrm{~s}$ and $1 \mathrm{~s}$ [1]. Magnetic random access memory (MRAM) is a solid-state-memory variant of the hard disk where the magnetic medium for storing and the magnetoresistive read element are merged into one. Unlike in hard disks, the magnetic stray field of the FM is not used for reading the FM bit and the latest spin-torque based MRAMs do not even use magnetic fields coupled to the FM moment for writing [2]. From this it appears natural to consider antiferromagnets (AFMs) as active building blocks of spintronic devices, where magnetic order is accompanied by a zero net magnetic moment [3-5].

Antiferromagnets are attractive for spintronics because they offer insensitivity to magnetic field perturbations, produce no perturbing stray fields, are readily compatible with metal, semiconductor, or insulator electronic structure, can act as a magnetic memory, and can generate large magnetotransport effects [6-8]. For example, two distinct stable states of an AFM with orthogonal AFM spin-axis directions were set in an FeRh Ohmic resistor and shown to be insensitive to fields as high as $9 \mathrm{~T}$ at ambient conditions [9]. A $~ 1 \%$ AFM anisotropic magnetoresistance (AMR) was used to electrically detect the states, in complete analogy to the $\sim 1 \%$ AMR of NiFeCo based bits in the first generation of FM MRAMs [10]. Also in analogy with the development of FM spintronics, very large $(\sim 100 \%)$ magnetoresistance signals were reported in AFM tunnel devices [7].

The AFM Néel-order spin-axis direction can be controlled indirectly by a magnetic field via an attached exchange-coupled FM [7,8] or, without the auxiliary FM, by techniques analogous to heat-assisted magnetic recording $[9,11]$. However, as with heat-assisted FM MRAMs $[12,13]$, the speed and energy efficiency of this method are limited. Here we predict a novel mechanism for AFM spin-axis reorientation by a lateral electrical current via Néel-order spin-orbit torque (NSOT) fields, i.e., via nonequilibrium fields that alternate in sign between the two spin sublattices. This relativistic mechanism does not involve FMs, heating, or magnetic fields, and offers ultrashort times unparalleled in FMs.

The microscopic origin of our NSOT fields is analogous to the relativistic spin-orbit torques (SOTs) observed recently in magnets with broken bulk or structural inversion symmetry [14-27], and is distinct in origin from the nonrelativistic spin-transfer torques $[3,28,29]$. We demonstrate below two types of NSOTs in two model systems.

A fieldlike NSOT appears in the $\mathrm{Mn}_{2} \mathrm{Au}$ AFM [4,30,31], whose $\mathrm{MoSi}_{2}$-type bct structure and AFM ordering are shown in Fig. 1(a). It is analogous to the fieldlike SOT arising from the inverse spin galvanic effect [14-22,25], observed previously in broken inversion-symmetry paramagnets or FMs. However, $\mathrm{Mn}_{2} \mathrm{Au}$ is bulk centrosymmetric and the current-induced NSOT arises from the fact that the 

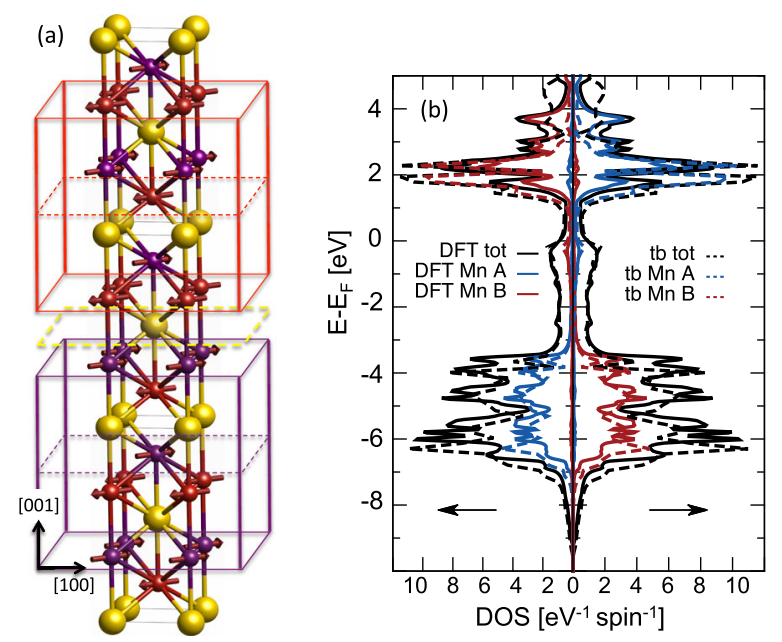

FIG. 1 (color online). (a) $\mathrm{Mn}_{2} \mathrm{Au}$ crystal structure and antiferromagnetic ordering. The two spin sublattices have broken inversion symmetry as illustrated by the red and purple colors. The full crystal is centrosymmetric around the $\mathrm{Au}$ atom as also highlighted in the figure. (b) Total, sublattice, and spin projected density of states from the $a b$ initio calculation and for the tightbinding Hamiltonian model.

lattice can be divided into two sublattices, which, individually, have broken inversion symmetry and form inversion partners [32]. Each sublattice gives opposite inverse spin galvanic effects, resulting in the NSOT field. The range of materials in which the relativistic current-induced torques can occur is therefore not restricted to FMs and, moreover, is not restricted to crystals with global broken inversion symmetry. In $\mathrm{Mn}_{2} \mathrm{Au}$, the inversion partner sublattices coincide with the two AFM spin sublattices, which makes the material an attractive candidate for observing the NSOT.

In AFMs where the two spin sublattices do not form inversion partners a NSOT can still occur. We illustrate it below in a 2D square lattice where the same broken inversion symmetry term in the Hamiltonian is shared by both spin sublattices. Here the resulting NSOT is analogous to the intrinsic antidamping SOT recently observed in broken bulk inversion symmetry FMs [27].

Models and methods. - $\mathrm{In} \mathrm{Mn}_{2} \mathrm{Au}$ we diagonalized a microscopic multiorbital tight-binding Hamiltonian to obtain the energy spectrum and eigenfunctions used in our transport calculations. The form of the tight-binding Hamiltonian was obtained following the procedure for bimetallic alloys described in Ref. [33]. The accuracy of the tight-binding energy spectrum is confirmed in Fig. 1(b) by comparing the electronic structure to the ab initio density-functional theory (DFT) calculations.

The other model structure comprises a 2D AFM square lattice with Rashba spin-orbit coupling due to the broken structural inversion symmetry and is relevant, e.g., to common experimental geometries in which a thin AFM film is interfaced with another layer. The model is sketched in Fig. 2(a) and its Hamiltonian is given by
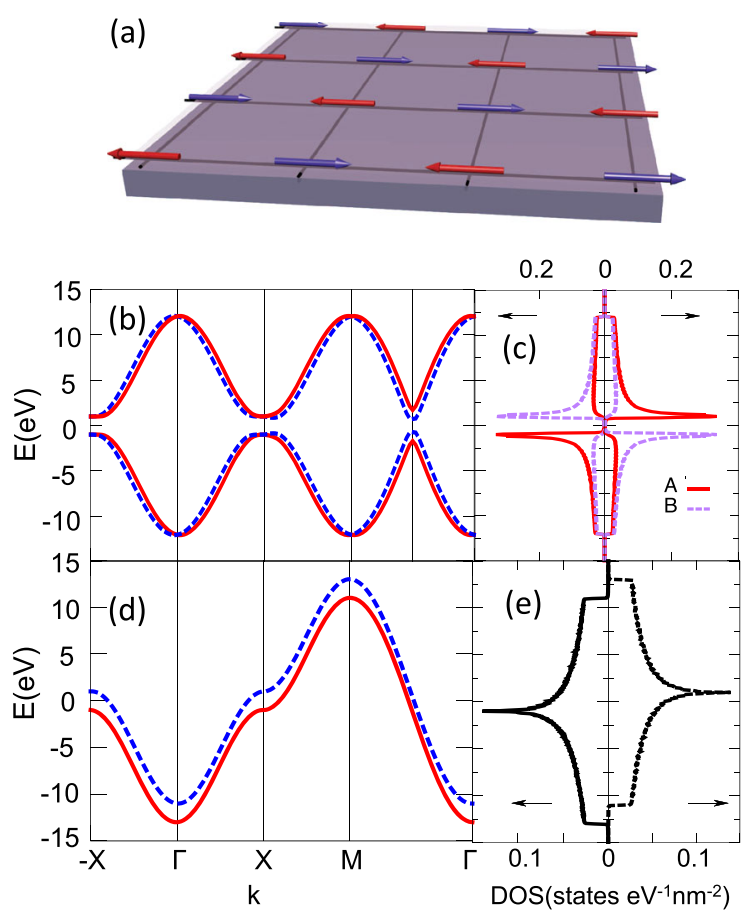

FIG. 2 (color online). (a) 2D AFM square lattice model with Rashba spin-orbit coupling. (b),(c) Band structure and the spinresolved density of states projected in each sublattice for the AFM state. (d),(e) Band structure and the spin-resolved density of states for the FM state. Here the hopping parameter $t_{N}=3.0 \mathrm{eV}$, $J_{s d}=1.0 \mathrm{eV}$, and $V_{S O}=0.1 \mathrm{eV}$.

$$
H=\sum_{\langle i j\rangle} J_{d d} \vec{S}_{i} \cdot \vec{S}_{j}+H^{\mathrm{tb}}+H_{R}+\sum_{i} J_{s d} \vec{s} \cdot \vec{S}_{i}
$$

Here $J_{d d}$ is the local moment (e.g., $d$ orbital) exchange constant, $J_{s d}$ is the local moment-carrier (e.g., $d$ and $s$ orbitals) exchange constant, $H^{\text {tb }}$ is the tight binding Hamiltonian for the carriers, and $H_{R}$ is the Rashba spinorbit interaction in a $2 \mathrm{D}$ system, given by

$$
\begin{aligned}
H_{R}= & V_{\mathrm{SO}} \sum_{i}\left[\left(c_{i \uparrow}^{\dagger} c_{i+\delta_{x} \downarrow}-c_{i \downarrow}^{\dagger} c_{i+\delta_{x} \uparrow}\right)\right. \\
& \left.-i\left(c_{i \uparrow}^{\dagger} c_{i+\delta_{y} \downarrow}+c_{i \downarrow}^{\dagger} c_{i+\delta_{y} \uparrow}\right)+\text { H.c. }\right],
\end{aligned}
$$

where $V_{\text {SO }}$ represents the spin-orbit coupling strength, and $\delta_{x}, \delta_{y}$ label the nearest neighbors direction.

The current-induced nonequilibrium spin density $\delta \vec{s}$ can be calculated via the Kubo linear response [19],

$$
\delta \vec{s}=\frac{\hbar}{2 \pi L^{2}} \operatorname{Re} \sum_{\vec{k} \alpha \beta}(\vec{s})_{\alpha \beta}(e \vec{E} \cdot \vec{v})_{\beta \alpha}\left[G_{\vec{k} \alpha}^{A} G_{\vec{k} \beta}^{R}-G_{\vec{k} \alpha}^{R} G_{\vec{k} \beta}^{R}\right],
$$

where the Green's functions are $\left.G_{\vec{k} \alpha}^{R}(E)\right|_{E=E_{F}} \equiv G_{\vec{k} \alpha}^{R}=$ $1 /\left(E_{F}-E_{\vec{k} \alpha}+i \Gamma\right)$, with the property $G^{A}=\left(G^{R}\right)^{*}$. Here, $L$ is the dimension of the 2D system, $e$ is the charge of electron, $\vec{E}$ is the applied electric field, $E_{F}$ is the Fermi energy, $E_{\vec{k} \alpha}$ is the energy spectrum, and $\Gamma$ is the spectral 
broadening that models the effect of disorder. For small $\Gamma$, we can separate the total $\delta \vec{s}$ into the intraband and interband contributions, with the intraband term given by

$$
\delta \vec{s}^{\text {intra }}=\frac{e E \hbar}{2 \Gamma} \int \frac{d^{3} k}{(2 \pi)^{3}} \sum_{\alpha}(\vec{s})_{\vec{k} \alpha}\left(v_{I}\right)_{\vec{k} \alpha} \delta\left(E_{\vec{k} \alpha}-E_{F}\right) .
$$

Here $(\vec{s})_{\vec{k} \alpha}$ denotes the expectation value of the carrier spin, and $\left(v_{I}\right)_{\vec{k} \alpha}$ the velocity component along the current direction. This intraband contribution in the Kubo formalism is equivalent to the Boltzmann transport theory expression [14-16,19,21] and, similar to the charge conductivity, $\delta \vec{s}^{\text {intra }} \sim 1 / \Gamma$.

The interband contribution dominating in the clean limit of $\Gamma \rightarrow 0$ is given by [19]

$$
\begin{aligned}
\delta s^{\text {inter }}= & \frac{\hbar}{L^{2}} \sum_{\vec{k} \alpha \neq \beta}\left(f_{\vec{k} \alpha}-f_{\vec{k} \beta}\right) \operatorname{Im}\left[(\vec{s})_{\alpha \beta}(e \vec{E} \cdot \vec{v})_{\beta \alpha}\right] \\
& \times \frac{\left(E_{\vec{k} \alpha}-E_{\vec{k} \beta}\right)^{2}-\Gamma^{2}}{\left[\left(E_{\vec{k} \alpha}-E_{\vec{k} \beta}\right)^{2}+\Gamma^{2}\right]^{2}} .
\end{aligned}
$$

Here, the labels $\alpha$ and $\beta$ correspond to different bands, and $f_{\vec{k} \alpha, \beta}$ is the Fermi distribution function.

Results in $\mathrm{Mn}_{2} \mathrm{Au}$. - In Figs. 3(b) and 3(c) we show the $\Gamma$-independent intraband NSOT field per applied current for $\mathrm{Mn}_{2} \mathrm{Au}$. It is evaluated from Eq. (4) and projected on each sublattice, assuming AFM spin-axis rotation in the [100][010] plane $(\phi=0$ corresponds to the [100] spin-axis direction) and in the [110]-[001] plane ( $\theta=0$ corresponds to the [110] easy-spin axis in $\mathrm{Mn}_{2} \mathrm{Au}$ ). Current is applied along the [100] direction and the NSOT field is obtained from the nonequilibrium spin density considering a typical exchangecoupling energy scale in transition metals $\sim 1 \mathrm{eV}$ [34].

NSOT fields on each sublattice are nonzero and have opposite sign. The largest component is in the [100]-[010] plane in the direction perpendicular to the applied current for all AFM spin-axis directions. The magnitude of the NSOT field in the $\mathrm{Mn}_{2} \mathrm{Au}$ AFM is comparable to the counterpart SOT fields observed in FM transition metal structures. Note that for current along the [001] direction the resulting NSOT field is zero.

The results imply that this intraband NSOT is an AFM counterpart of the inverse spin galvanic effect [35], or the intraband, fieldlike, SOT [14-22,25], observed previously in broken inversion-symmetry, spin-orbit coupled paramagnets or FMs. We illustrate in Fig. 3(a) how these current induced nonequilibrium fields arise in structures with broken inversion symmetry. Here we choose the case of a Rashba spin-orbit coupled 2D system for simplicity. The electric field induces an asymmetric nonequilibrium distribution function of carrier eigenstates and as a result a net polarization ensues that depends on the scattering time, hence its link to extrinsic scattering origin. In magnets, the nonequilibrium carrier spin density acts on magnetic
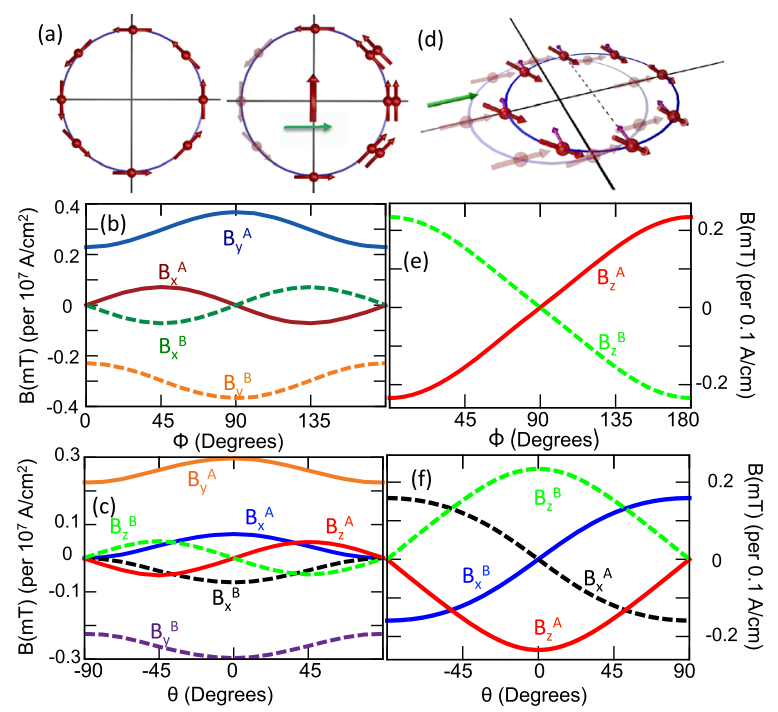

FIG. 3 (color online). (a) Schematics of the intraband, inverse spin galvanic effect in a model Rashba system. The left panel represents the equilibrium distribution of spins (red arrows); the right panel shows the nonequilibrium redistribution resulting in a net in-plane spin polarization (thick red arrow) perpendicular to the current (green arrow). (b) Intraband NSOT field in $\mathrm{Mn}_{2} \mathrm{Au}$ as a function of the in-plane spin-axis angle. The sublattice index $A$ or $B$ and component of the field $x, y$, or $z$ ([100], [010], [001]) are shown for each curve. (c) Same as (b) for the out-of-plane spinaxis angle. (d) Schematics of the intrinsic interband contribution to the nonequilibrium spin polarization. In equilibrium all spins are approximately aligned with the exchange field, which is considered to be stronger than the Rashba field. A nonequilibrium in-plane Rashba field (purple arrows) aligned perpendicular to the applied current causes an out-of-plane tilt of the carrier spins on the shifted Fermi surface. (e),(f) Interband NSOT fields as a function of spin-axis angles in the 2D Rashba AFM for $\Gamma=0.01 \mathrm{eV}$ and $E_{F}=-2 \mathrm{eV}$. Other parameters of the model are as in Fig. 2 In all panels the current is along the [100] axis.

moments as an effective magnetic field when carrier spins are exchange coupled to the magnetic moments.

The full lattice of the $\mathrm{Mn}_{2} \mathrm{Au}$ crystal has an inversion symmetry and the first expectation would be that there is no current-induced spin density. However, the lattice is formed by two sublattices, which, individually, have broken inversion symmetry and form inversion partners along the [001] axis. These coincide with the spin sublattices of the AFM ground state in $\mathrm{Mn}_{2} \mathrm{Au}$, as highlighted in Fig. 1(a). The two sublattices forming the inversion partners in the $\mathrm{Mn}_{2} \mathrm{Au}$ crystal are at the origin of the observed intraband NSOT.

Results in the model 2D Rashba AFM.-Since both spin sublattices experience the same inversion symmetry breaking Rashba field in our 2D AFM model, the intraband contribution to the current induced spin polarization has the same sign on both spin sublattices, i.e., is not staggered. A NSOT field is found, however, when evaluating the interband term $\delta \vec{s}^{\text {inter }}$ from Eq. (5). The Néel-order currentinduced field components projected on each sublattice are shown in Figs. 3(e) and 3(f) for the AFM spin-axis rotation 
in the [100]-[010] plane and in the [100]-[001] plane. Here, we plot the corresponding NSOT field per applied current along the [100] direction.

The interband contribution described by Eq. (5) arises from the time-dependent quantum-mechanical perturbation of the eigenstates between collisions [illustrated in Fig. 3(d)] and is the basis of the Berry curvature mechanism introduced to explain the intrinsic anomalous Hall effect [36], the intrinsic spin-Hall effect [37], and most recently also the intrinsic antidamping spin-orbit torque in FMs $[27,38]$. The key phenomenology that distinguishes the interband antidamping spin-orbit field from the intraband field is the harmonic dependence on the in-plane and out-of-plane spin-axis angles, as shown in Figs. 3(e) and 3(f). Another important feature, illustrated in Figs. 4(a) and 4(b), is that the interband NSOT in the AFM can be significantly larger than its FM SOT counterpart. The interband nature of the term $\delta \vec{s}^{\text {inter }}$ from Eq. (5) implies that its magnitude is large when two subbands linked by spin-orbit coupling have a small energy spacing. In the calculations shown in Figs. 3(e) and 3(f) and Figs. 4(a) and 4(b) for the model 2D lattice, we consider weak spin-orbit coupling relative to the exchange energy strength [27]. The smallest band splitting in this limit is governed by the exchange energy in the FM state while in the AFM state it is given by the small spin-orbit splitting. The band structures with their corresponding splittings are plotted in Fig. 2 together with the corresponding densities of states (DOSs) showing the characteristic van Hove singularities of the 2D square lattice. As shown in Figs. 4(a) and 4(b), the interband current induced spin-orbit fields are
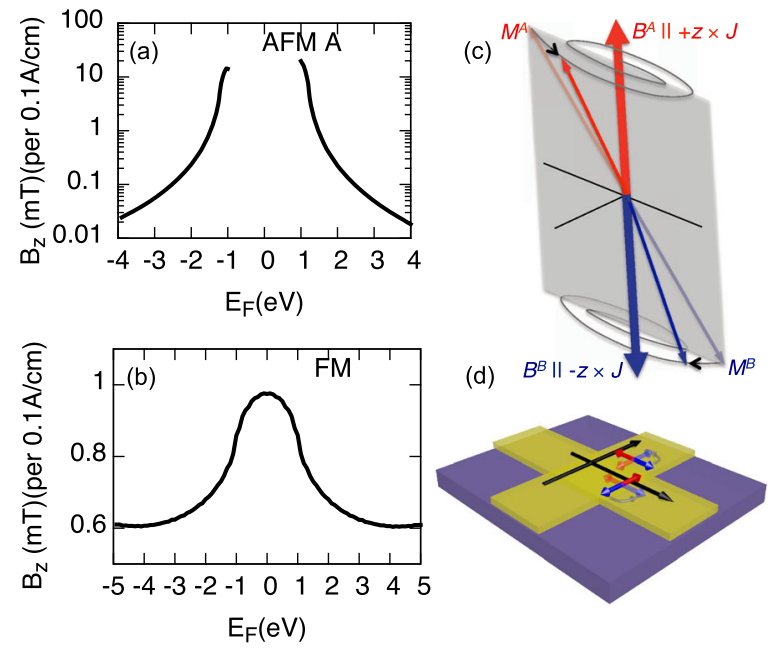

FIG. 4 (color online). (a) The $z$ component of the NSOT field in the 2D square-lattice Rashba AFM for $\phi=0$ as a function of the Fermi energy. (b) Same as (a) for the FM state of the 2D Rashba square lattice. (c) Schematics of the AFM dynamics in a staggered field generating the fieldlike torque. (d) Schematics of the electrical writing scheme via the NSOT in a memory device built in an AFM with cubic easy axes. Black arrows represent the cross-wire writing currents and either of the current lines can be also used for reading by the AMR. Red-blue arrows show the current-induced AFM spin-axis reorientation. enhanced in the vicinity of the DOS singularities in both the FM and AFM states. However, in the AFM state with the small energy spacing of the two spin-orbit coupled bands, the enhancement is much larger, reaching 3 orders of magnitude in the present calculations.

Discussion.-In our 2D Rashba model we identified a relativistic microscopic mechanism by which an electrical current $\mathbf{J}$ driven in a plane of an AFM layer with broken space-inversion symmetry generates an antidamping NSOT. It acts on the sublattice magnetizations $\mathbf{M}^{A}=$ $-\mathbf{M}^{B}$, in the form $\mathbf{T}^{A / B} \sim \mathbf{M}^{A / B} \times \mathbf{B}^{A / B}$, where $\mathbf{B}^{A / B} \sim$ $\left[\mathbf{M}^{A / B} \times(\hat{z} \times \mathbf{J})\right]$. The effective field $\mathbf{B}^{A / B}$ is staggered in this case due to the opposite magnetizations on the two sublattices. This is reminiscent of an earlier phenomenological prediction of a nonrelativistic antidamping spin transfer torque (STT) generated in an AFM by an effective field $\mathbf{B}^{A / B} \sim \mathbf{M}^{A / B} \times \mathbf{p}_{\mathrm{FM}}$ due to a vertical spin current from an adjacent FM layer polarized along a vector $\mathbf{p}_{\mathrm{FM}}$ [39]. A detailed study of the corresponding Landau-Lifshitz-Gilbert dynamics (assuming a fixed $\mathbf{p}_{\mathrm{FM}}$ ) showed that above a critical current, at which the energy loss due to internal damping is compensated by the current-induced pumping, the configuration of $\mathbf{M}^{A / B} \| \mathbf{p}_{\mathrm{FM}}$ becomes unstable and is switched to a stable $\mathbf{M}^{A / B} \perp \mathbf{p}_{\mathrm{FM}}$ state [39]. Because this is independent of the sign of the vertical current, the STT cannot switch the AFM back to the $\mathbf{M}^{A / B} \| \mathbf{p}_{\mathrm{FM}}$ configuration. In the case of our antidamping NSOT, $\mathbf{p}_{\mathrm{FM}}$ is replaced with $\hat{z} \times \mathbf{J}$ and a reversible $90^{\circ}$ reorientation of the AFM can be achieved by redirecting the in-plane current $\mathbf{J}$ between two orthogonal directions. Note that this favorable property of an antidamping torque induced in the AFM by an in-plane current would apply not only to our relativistic NSOT but also to a spin injection into the AFM from an adjacent paramagnet layer via the relativistic spin Hall effect $[23,24]$.

The fieldlike STT acting on the AFM in the FM-AFM bilayer has the form $\mathbf{T}^{A / B} \sim \mathbf{M}^{A / B} \times \mathbf{B}^{A / B}$ with $\mathbf{B}^{A / B} \sim$ $\mathbf{p}_{\mathrm{FM}}$; i.e., $\mathbf{B}^{A / B}$ does not have the desired staggered property [29]. This illustrates why fieldlike nonrelativistic STTs have been neglected in the Landau-Lifshitz-Gilbert dynamics induced by an electrical current in AFMs. In our microscopic study of the $\mathrm{Mn}_{2} \mathrm{Au} \mathrm{AFM}$ we have demonstrated, however, that a fieldlike torque $\mathbf{T}^{A / B} \sim \mathbf{M}^{A / B} \times \mathbf{B}^{A / B}$ with a staggered $\mathbf{B}^{A} \sim+\hat{z} \times \mathbf{J}$ and $\mathbf{B}^{B} \sim-\hat{z} \times \mathbf{J}$ can be generated by current in special crystal structures in which the AFM spin sublattices coincide with the two inversion-partner sublattices. The corresponding dynamics comprising (damped) elliptical precessional motion with opposite helicities of the two spin sublattices is sketched in Fig. 4(c). For a detailed description of these modes we refer to Eq. (9) and the corresponding discussion in Ref. [40]. Again, as in the antidamping NSOT case, a cross-wire geometry can be used to reversibly switch between two orthogonal AFM spin-axis directions using the fieldlike NSOT. For the theoretical (001)-plane anisotropy energies in $\mathrm{Mn}_{2} \mathrm{Au}$ [4], our estimates of the NSOT fields suggest sizable reorientations at current densities $\sim 10^{8}-10^{9} \mathrm{~A} / \mathrm{cm}^{2}$, depending on the angle of the 
applied in-plane current with respect to the easy and hard anisotropy axes.

Ultrafast (ps-scale) reorientation of the AFM spin axis in a staggered field was previously reported in an optical pumpand-probe study of $\mathrm{AFM} \mathrm{TmFeO}{ }_{3}$ [41]. The origin of the staggered field was different than considered here; the material had a temperature dependent AFM easy-axis direction and the corresponding Néel-order anisotropy field was induced by laser heating the sample above the easy-axis transition temperature. The microscopic origin of the staggered field is not crucial, however, for the time scale of the spin dynamics, which in AFMs is typically 2-3 orders of magnitude shorter than in FMs [40]. We can therefore infer from these experiments that the AFM spin-axis reorientation due to our current-induced NSOT will not be limited by the ultrafast AFM spin dynamics itself but only by the circuitry time scales for delivering the electrical pulses, which can be of the order of $\sim 100 \mathrm{ps}$ [42]. A schematic of a device that can be used to reverse the AFM spin axis electrically between two orthogonal directions is shown in Fig. 4(d). In a cross-wire geometry, each wire stabilizes via the NSOT one of the two orthogonal spin-axis directions. An AFM AMR [8,9] measured in one of the arms can then be used to electrically detect the spin-axis direction. In an AFM with cubic magnetic anisotropy, an all-electrical AFM memory device can be realized based on this scheme.

We acknowledge fruitful discussions with R. Campion, K. Edmonds, A. Ferguson, B. Gallagher, X. Marti, V. Novak, K. Olejnik, H. Reichlova, A. Rushforth, H. Saidaoui, and P. Wadley, and support from the EU European Research Council (ERC) Advanced Grant No. 268066, the Ministry of Education of the Czech Republic Grant No. LM2011026, the Grant Agency of the Czech Republic Grant No. 14-37427G, the Academy of Sciences of the Czech Republic Praemium Academiae, the NSF Grant No. DMR-1105512, the ONR Grant No. 141110780, and the Alexander Von Humboldt Foundation. A. M. was supported by the King Abdullah University of Science and Technology. J.Z. and H. G. contributed equally to this work.

[1] C. Chappert, A. Fert, and F. N. V. Dau, Nat. Mater. 6, 813 (2007).

[2] http://www.everspin.com/PDF/ST-MRAM_Technical_Brief .pdf.

[3] A. S. Núñez, R. A. Duine, P. Haney, and A. H. MacDonald, Phys. Rev. B 73, 214426 (2006).

[4] A. B. Shick, S. Khmelevskyi, O. N. Mryasov, J. Wunderlich, and T. Jungwirth, Phys. Rev. B 81, 212409 (2010).

[5] T. Jungwirth et al., Phys. Rev. B 83, 035321 (2011).

[6] I. V. Shvets, A. N. Grigorenko, K. S. Novoselov, and D. J. Mapps, Appl. Phys. Lett. 86, 212501 (2005).

[7] B. G. Park et al., Nat. Mater. 10, 347 (2011).

[8] I. Fina et al., Nat. Commun. 5, 4671 (2014).

[9] X. Marti et al., Nat. Mater. 13, 367 (2014).

[10] J. M. Daughton, Thin Solid Films 216, 162 (1992).

[11] D. Petti et al., Appl. Phys. Lett. 102, 192404 (2013).
[12] E. Fullerton, S. Maat, and J. U. Thiele, U.S. Patent No. 20050281081 (2005).

[13] B. Dieny et al., Int. J. Nanotechnology 7, 591 (2010).

[14] B. A. Bernevig and O. Vafek, Phys. Rev. B 72, 033203 (2005).

[15] A. Manchon and S. Zhang, Phys. Rev. B 78, 212405 (2008).

[16] A. Manchon and S. Zhang, Phys. Rev. B 79, 094422 (2009).

[17] A. Matos-Abiague and R. L. Rodriguez-Suarez, Phys. Rev. B 80, 094424 (2009).

[18] A. Chernyshov, M. Overby, X. Liu, J. K. Furdyna, Y. LyandaGeller, and L. P. Rokhinson, Nat. Phys. 5, 656 (2009).

[19] I. Garate and A. H. MacDonald, Phys. Rev. B 80, 134403 (2009).

[20] M. Endo, F. Matsukura, and H. Ohno, Appl. Phys. Lett. 97, 222501 (2010).

[21] D. Fang, H. Kurebayashi, J. Wunderlich, K. Výborný, L. P. Zârbo, R. P. Campion, A. Casiraghi, B. L. Gallagher, T. Jungwirth, and A. J. Ferguson, Nat. Nanotechnol. 6, 413 (2011).

[22] I. M. Miron, G. Gaudin, S. Auffret, B. Rodmacq, A. Schuhl, S. Pizzini, J. Vogel, and P. Gambardella, Nat. Mater. 9, 230 (2010).

[23] I. M. Miron, K. Garello, G. Gaudin, P.-J. Zermatten, M. V. Costache, S. Auffret, S. Bandiera, B. Rodmacq, A. Schuhl, and P. Gambardella, Nature (London) 476, 189 (2011).

[24] L. Liu, C.-F. Pai, Y. Li, H. W. Tseng, D. C. Ralph, and R. A. Buhrman, Science 336, 555 (2012).

[25] H. Li, X. Wang, F. Dogan, and A. Manchon, Appl. Phys. Lett. 102, 192411 (2013).

[26] K. Garello, I. M. Miron, C. O. Avci, F. Freimuth, Y. Mokrousov, S. Blügel, S. Auffret, O. Boulle, G. Gaudin, and P. Gambardella, Nat. Nanotechnol. 8, 587 (2013).

[27] H. Kurebayashi et al., Nat. Nanotechnol. 9, 211 (2014).

[28] D. C. Ralph and M. D. Stiles, J. Magn. Magn. Mater. 320, 1190 (2008).

[29] E. V. Gomonay and V. M. Loktev, Low Temp. Phys. 40, 17 (2014).

[30] H. Wu, Z.-M. Liao, R. G. Sumesh Sofin, G. Feng, X.-M. Ma, A. B. Shick, O. N. Mryasov, and I. V. Shvets, Adv. Mater. 24, 6374 (2012).

[31] V. M. T. S. Barthem, C. V. Colin, H. Mayaffre, M. Julien, and D. Givord, Nat. Commun. 4, 2892 (2013).

[32] X. Zhang, Q. Liu, J.-W. Luo, A. J. Freeman, and A. Zunger, Nat. Phys. 10, 387 (2014).

[33] J. Zemen, J. Mašek, J. Kučera, J. A. Mol, P. Motloch, and T. Jungwirth, J. Magn. Magn. Mater. 356, 87 (2014).

[34] P. M. Haney, H. W. Lee, K. J. Lee, A. Manchon, and M. D. Stiles, Phys. Rev. B 88, 214417 (2013).

[35] V. M. Edelstein, Solid State Commun. 73, 233 (1990).

[36] N. Nagaosa, J. Sinova, S. Onoda, A. H. MacDonald, and N. P. Ong, Rev. Mod. Phys. 82, 1539 (2010).

[37] T. Jungwirth, J. Wunderlich, and K. Olejnik, Nat. Mater. 11, 382 (2012).

[38] F. Freimuth, S. Blügel, and Y. Mokrousov, arXiv:1305.4873.

[39] H. V. Gomonay and V. M. Loktev, Phys. Rev. B 81, 144427 (2010).

[40] F. Keffer and C. Kittel, Phys. Rev. 85, 329 (1952).

[41] A. V. Kimel, A. Kirilyuk, A. Tsvetkov, R. V. Pisarev, and Th. Rasing, Nature (London) 429, 850 (2004).

[42] H. W. Schumacher, C. Chappert, P. Crozat, R. Sousa, P. Freitas, J. Miltat, J. Fassbender, and B. Hillebrands, Phys. Rev. Lett. 90, 017201 (2003). 